\title{
Single institute experience of pancreatico-enteric anastomosis failure after pancreaticoduodenectomy
}

Yoonkyung Woo, Youngkyoung You, Jaehyun Han, Hojoong Choi, Yumi Kim, Bongjun Kwak, Taeho Hong, Donggoo Kim

Department of Surgery, Seoul St. Mary's Hospital, College of Medicine, The Catholic University of Korea, Seoul, Korea

Purpose: We have summarized the experience of our institution related to what treatment has been performed in patients with pancreatic fistula and their outcome.

Methods: Seventy-eight pancreatico-enteric anastomosis failure (PEAF) patients of 403 pancreaticoduodenectomy (PD) were included for this retrospective study. PEAF was defined by the presence of rich amylase (over 10,000 IU/L) in drainage fluid at postoperative day 5 to 7 and radiographic demonstration of the anastomotic breakdown and associated local fluid collection. The management was analyzed by observation group (0 group), intervention (I group) and surgery group (S group).

Results: Preoperative clinical status of the PEAF group and non-PEAF group was similar. Bile duct cancer was the highest risk subgroup of the PEAF $(P=0.001)$ and the pancreatic adenocarcinoma showed the least risk for the PEAF $(P<0.001)$. Among the 78 PEAF patients, 50 were managed as a conservative treatment, 15 patients were received radiologic intervention and 13 patients performed rescue surgery. Among these three subgroups, there was no statistical significance in the patient's demographics, clinical status, surgical factors and disease nature. However, mortality was significantly higher in the $\mathrm{S}$ group $(\mathrm{P}<0.001)$. The mortality cases were developed one and six patients in 0 and $\mathrm{S}$ group, respectively. Surgical procedures in $S$ group were completion total pancreatectomy with or without splenectomy $(n=12)$ and pancreatectomy preserving spleen in four (28.6\%). Pancreaticogastrostomy repair and Roux-en-Y pancreaticojejunostomy reconstruction were performed each case, respectively.

Conclusion: Proper drainage catheter indwelling during the PD or postoperative radiological intervention can effectively manage the PEAF without surgical interventional treatment.

Keywords: Pancreaticoduodenectomy, Pancreaticojejunostomy, Pancreaticogastrostomy, Pancreatic fistula

\section{INTRODUCTION}

Pancreaticoduodenectomy (PD) is a surgical procedure for the pathologic lesion of pancreas head and duodenum, periampullary area, regardless of benign and malignant disease. This procedure has been associated with a significant risk of morbidity and mor-

Received: May 29, 2019 Revised: Jun 18, 2019 Accepted: Jun 20, 2019 Correspondence to: Youngkyoung You

Department of Surgery, Seoul St. Mary's Hospital, College of Medicine,

The Catholic University of Korea, 222 Banpo-daero, Seocho-gu, Seoul

06591, Korea

Tel: +82-2-2258-2876, Fax: +82-2-595-2822

E-mail: yky602@catholic.ac.kr

ORCID: Yoonkyung Woo (https://orcid.org/0000-0001-6423-834X), Youngkyoung You (https://orcid.org/0000-0002-8506-0752)

Copyright @ 2019 Korean Society of Surgical Oncology

This is an Open Access article distributed under the terms of the Creative Commons Attribution Non-Commercial License (http://creativecommons.org/licenses/by-nc/4.0) which permits unrestricted non-commercial use, distribution, and reproduction in any medium, provided the original work is properly cited. tality. Recently, the mortality rate associated with PD has been decreased to less than $5 \%$, thanks to the improvement of surgical skills and perioperative management. But the morbidity rate still remains high, ranging from $30 \%$ to $50 \%$ [1-3]. The most prevalent factor to lead the mortality following PD is a postoperative pancreatic fistula. Pancreatic fistula is an abnormal communication between the pancreas and other organs due to leakage of pancreatic secretions from damaged pancreatic ducts. Followed by pancreatic fistula, some complications are coincided not uncommon, such as bleeding, delayed gastric emptying, intraabdominal abscess, wound infection, etc. $[4,5]$.

For reduce the incidence rate of pancreatico-enteric anastomosis failure (PEAF), various surgical methods of pancreas reconstruction have been proposed. Most pancreas reconstruction was made as pancreaticojejunostomy (PJ) and pancreaticogastrostomy (PG). Some surgeon prefers PG which has been known to have advantage in the anastomosis failure [6]. However, it is still controversial whether a method is more effective in preventing pancreatic fistula 
Table 1. Definition of pancreatico-enteric anastomosis failure (PEAF)

\begin{tabular}{ll}
\hline Amylase & $>10,000 \mathrm{IU} / \mathrm{L}$ \\
Postoperative time & $>5$ Days \\
Radiographic feature & $\begin{array}{c}\text { Anastomotic breakdown associated with local fluid } \\
\text { collection }\end{array}$ \\
\hline
\end{tabular}

$[7,8]$.

Early diagnosis and prevention of pancreatic fistula have been discussed, but the treatment is still unclear. We have focused on the leakage from the enteric anastomosis of pancreas and called PEAF, among the broad concept of fistula. And we have summarized the experience of our institution related to what treatment has been performed in patients with PEAF and their outcome in this study (Table 1).

\section{METHODS}

From March 2010 to May 2017, 403 patients with periampullary lesions included disease of pancreas head, duodenum, and distal bile duct underwent classical PD and pylorus preserving pancreaticoduodenectomy. Patients undergoing other types of pancreatic resections were excluded. All surgical procedures were performed by three specialized surgeons (YY, TH, and DK) at our institute. Reviewed data were included patient demographics (age, sex, and diagnosis, American Society of Anesthesiologists [ASA] classification), type of operation (laparoscopic or laparotomic), mortality and the timing of procedure. Postoperative mortality was defined as death within postoperative 2 month after PD.

Data for all recruited cases are retrospectively reviewed. And all data were approved by the Institutional Review Board of Seoul St. Mary's Hospital (IRB No. KC18RCSI0043). The informed consent was waived.

PEAF was defined by the presence of rich amylase (over 10,000 $\mathrm{IU} / \mathrm{L}$ ) in drainage fluid at postoperative day 5 to 7 . Also, it is included that cases of suspicious PEAF of radiographic demonstration by computed tomography (CT) such as showing the anastomotic breakdown and associated local fluid collection (Table 1).

According to this definition, of the 403 patients who underwent PD, postoperative PEAF developed in 78 patients (19.4\%). And they were categorized into three groups according to the post PEAF management $[9,10]$. Those are as follows; (1) Observation group (O group): they were managed by conservative treatment such as maintaining drainage catheter indwelling during the PD, proper nutrition support by enteral feeding with or without total parenteral nutrition and prophylactic antibiotics coverage. (2) Intervention group (I group): they were performed postoperative ra- diologic intervention for PEAF management. (3) Surgery group (S group): they were received re-laparotomy for surgical drainage and more definitive intervention. Decision of treatment was depended on the patient vital sign and status. Even the patient meets the criteria of PEAF, most of the patients were included in the $\mathrm{O}$ group in the stable vital sign and no local inflammatory findings. Otherwise intervention was considered first in the exception of the surgeon's subjective opinion based on operation findings basically according to the remnant pancreas consistency. Also failed radiologic interventional attempts were the indications of the reoperation. In this series, 16 patients died in the entire patient group and seven cases were related to PEAF.

\section{Surgical techniques}

Pancreatico-enteric anastomosis methods are basically performed by PJ and PG anastomosis. First of all, end-to-end PJ (dunking method) performed for the anastomosis of pancreatic remnant and jejunum by the three surgeons. Based on these, we modified it according to case in considering of pancreas consistency and patient's general condition. In the case of soft pancreas and poor general condition, PG was preferred to PJ in our series. PJ and PG anastomosis was performed as described in the report by Batignani et al. [11], Kim et al. [12], and Hong et al. [13]. Of 403 PD cases, 341 patients underwent laparotomic approach, on the other hand, 62 underwent laparoscopic approach. Also, PJ was performed in 210 patients, PG was performed to 193 patients. The decision making about proper surgical approach was based on patient's pancreas condition and general condition as described above. So, we preferred PG if the texture of the pancreas was softer. If the pancreatic duct was dilated more than $5 \mathrm{~mm}$, end-to-side duct-to-mucosa anastomosis was performed in selectively. Two or three closed suction drains (Jackson-Pratt drain) were placed in all patients, near biliary-enteric and pancreatico-enteric anastomosis.

\section{Statistical methods}

Data analyses were performed using IBM SPSS version 24.0 (IBM Corp., Armonk, NY, USA). Univariate analysis was performed using Pearson chi-square test for categorical data using frequency distribution and percentages. For continuous variables, the student $\mathrm{t}$-test was used to compare the outcomes between the two groups, and Kruskal-Wallis test was performed to compare the results among three groups. The $95 \%$ confidence interval of the difference in proportions was calculated. Descriptive statistics were recorded as the means \pm standard deviation. Under 0.05 of P-value was considered statistically significant. 


\section{RESULTS}

There were 231 male patients (57.3\%) and 172 female patients (42.7\%) with a mean age of $64.4 \pm 11.2$ years. When classified according to ASA classification, 47,327, and 29 patients were class I, II, and III. As surgical procedure in terms of pancreatico-enteric anastomosis, the laparotomic PJ was performed in 191 (47.4\%), laparotomic PG was in 150 (37.2\%), laparoscopic PG was in 43 (10.7\%) and laparoscopic PJ was in 19 (4.7\%).

In pathologic diagnosis, 342 cases were malignant diseases. The most common malignant disease was pancreas cancer $(n=153$, $44.7 \%$ of the malignant diseases) and distal bile duct cancer was 97 cases (28.4\%). Ampulla of Vater cancer and pancreas neuroendocrine tumor were 69 cases (20.2\%) and nine cases (2.6\%) respectively. Duodenal adenocarcinoma and duodenal malignant lymphoma were eight cases (2.3\%). Pancreas metastasis of other cancer was five cases, gallbladder adenocarcinoma as two cases, respec-

Table 2. Demographics of patients who underwent pancreatico-duodenectomy in the Catholic University Seoul St. Mary's Hospital (from 2013 to 2017)

\begin{tabular}{lc}
\hline Characteristic & Total $(\mathrm{n}=403)$ \\
\hline Age (yr) & $64.4 \pm 11.2$ \\
Sex (male:female) & $231: 172$ \\
ASA class & \\
I & $47(11.7)$ \\
II & $327(81.1)$ \\
III \& IV & $29(7.2)$ \\
Type of anastomosis & \\
Laparoscopic & 62 \\
PJ & $19(4.7)$ \\
PG & $43(10.7)$ \\
Laparotomic & 341 \\
PJ & $191(47.4)$ \\
PG & $150(37.2)$ \\
Pathology & \\
Benign & \\
Malignant & $61(15.1)$ \\
Pancreas adenocarcinoma & $342(84.9)$ \\
Bile duct adenocarcinoma & 153 \\
Ampulla of Vater adenocarcinoma & 97 \\
Pancreatic neuroendocrine tumor & 69 \\
Duodenal neoplasm & 9 \\
Other cancer pancreas metastasis & 8 \\
Gallbladder cancer & 4 \\
\hline
\end{tabular}

Values are presented as mean \pm standard deviation or number (\%).

ASA, American Society of Anesthesiologists; PJ, pancreaticojejunostomy; PG, pancreaticogastrostomy.

${ }^{a}$ Benign disease: pancreatic cystic neoplasm, pancreatitis, pancreas injury and duodenal tumor, duodenal perforation, periampullary adenoma, etc. tively. The remaining benign diseases included pancreatic cystic neoplasm, pancreatitis, pancreas injury and duodenal tumor, duodenal perforation, periampullary adenoma and etc. were 61 cases (15.1\%) (Table 2).

PEAF was developed in 78 from 403 patients (19.4\%). In comparing between contrast group (non-PEAF group) and PEAF developed group (PEAF group), the mean age of both group was similar, but the PEAF incidence was significantly higher in the male sex $(\mathrm{P}=0.009)$. Neither ASA score nor pancreatico-enteric anastomosis type affected PEAF incidence rate. In terms of disease entity, bile duct cancer was the highest risk disease subgroup of the PEAF ( $\mathrm{P}=0.001)$ which was 30 out of 97 cases and the benign disease had reluctance to develop PEAF than the other indication of surgery $(\mathrm{P}=0.056)$. And also, in the nine pancreatic neuroendocrine tumor cases, four patients (44.4\%) suffered from the PEAF, but it has not significant statistically $(\mathrm{P}=0.075)$. On the other hand, PEAF was developed in 14 of 153 pancreatic adenocarcinoma therefore it was the least risk for the PEAF $(\mathrm{P}<0.001)$. The mean age of patients with benign disease in PEAF was 58 , which was significantly lower than that of the entire PEAF group. The mean age of patients with malignant disease was 63 years old (Table 3 ).

Among the 78 PEAF patients, 50 was managed as a conservative treatment (O group) that was consisted with maintenance of drain, enteral feeding with or without parenteral nutritional support and prophylactic antibiotics. Fifteen patients of PEAF were successfully treated with radiologic intervention (percutaneous transabdominal drainage procedure) (I group). Surgery group was consisted with 13 PEAF patients (S group) who included one failed intervention case. Among three groups, there was no statistical significance in the patient's demographics, clinical status (ASA classification), surgical factors and disease nature. However, mortality was significantly higher in the $S$ group $(\mathrm{P}<0.001)$. The mortality cases were developed one and six patients in $\mathrm{O}$ and $\mathrm{S}$ group, respectively. Even if the multiple interventions or surgical procedures were performed in one patient, each procedure was separately counted. As a result, the mean interval from the $\mathrm{PD}$ to procedures were 17 days (range, 6-34 days) in I group and 12.2 days (range, 1-27 days) in S group (Table 4).

The most common surgical procedure in $S$ group was completion total pancreatectomy with splenectomy, and it was conducted 8 out of 14 rescue-operation (57.1\%). The next was completion total pancreatectomy preserving spleen in four (28.6\%). PG repair and Roux-en-Y PJ reconstruction were performed in each case, respectively. In one patient, reoperation and re-reoperations were performed as Roux-en-Y PJ repair and completion total pancreatectomy with splenectomy, and the patient survived. The mean duration from PD to relaparotomy was variable, completion total 
Table 3. Comparison of demographics between patients of non-PEAF group and the the group PEAF developed $(n=403)$

\begin{tabular}{|c|c|c|c|}
\hline Characteristic & Non-PEAF $(n=325)$ & $\operatorname{PEAF}(n=78)$ & P-value \\
\hline Age (yr) & $64.9 \pm 10.7$ & $62.0 \pm 12.7$ & 0.37 \\
\hline Sex (male:female) & $176: 149$ & $55: 23$ & 0.009 \\
\hline \multicolumn{4}{|l|}{ ASA class } \\
\hline I & $37(11.4)$ & $10(12.8)$ & 0.782 \\
\hline$\|$ & $266(81.8)$ & $61(78.2)$ & 0.460 \\
\hline III \&IV & $22(6.8)$ & $7(9.0)$ & 0.433 \\
\hline \multicolumn{4}{|l|}{$\begin{array}{l}\text { Type of pancreatico-enteric anastomosis } \\
\text { Laparoscopic }\end{array}$} \\
\hline PJ & 18 & 1 & 0.218 \\
\hline$P G$ & 31 & 12 & 0.133 \\
\hline \multicolumn{4}{|l|}{ Laparotomic } \\
\hline PJ & 119 & 41 & 0.333 \\
\hline$P G$ & 157 & 24 & 0.206 \\
\hline \multicolumn{4}{|l|}{ Pathology } \\
\hline Benign $^{\text {a) }}$ & 43 & 18 & 0.056 \\
\hline Malignant & 282 & 60 & 0.029 \\
\hline Pancreas adenocarcinoma & 139 & 14 & $<0.001$ \\
\hline Bile duct adenocarcinoma & 67 & 30 & 0.001 \\
\hline Ampulla of Vater adenocarcinoma & 57 & 12 & 0.650 \\
\hline Pancreatic neuroendocrine tumor & 5 & 4 & 0.075 \\
\hline Duodenal neoplasm & 8 & 0 & 0.363 \\
\hline Other cancer pancreas metastasis & 4 & 0 & 1.00 \\
\hline Gallbladder cancer & 2 & 0 & 1.00 \\
\hline
\end{tabular}

Values are presented as mean \pm standard deviation or number (\%).

PEAF, pancreatico-enteric anastomosis failure; ASA, American Society of Anesthesiologists; PJ, pancreaticojejunostomy; PG, pancreaticogastrostomy.

a) Benign disease: pancreatic cystic neoplasm, pancreatitis, pancreas injury and duodenal tumor, duodenal perforation, periampullary adenoma, etc.

pancreatectomy with splenectomy was 11.4 days (range, 1-26 days) and the mean time to the completion total pancreatectomy preserving spleen was 16.7 days (range, 1-27 days) (Table 5).

One in 13 patients in the $S$ group received intervention but failed and underwent rescue operation. We performed coil embolization for superior mesenteric artey bleeding and succeeded in bleeding control. However, as the symptoms and signs of peritonitis such as leukocytosis and mild fever, abdominal pain persisted in this patient, we decided to do surgery. This is probably due to the fact that the inflammation has already progressed. The remaining 12 patients underwent rescue operation immediately without intervention, for two main reasons. The first is the case that the patient is older or has other comorbid diseases and so there is a concern for fatal progress, if intervention is attempted and failed. And, the second is the case that vital sign is unstable, such as fever, tachycardia, or hypotension. When localized abdominal inflammation progressed to pan peritonitis and generalized inflammation, in other words, sepsis was suspected, surgery was performed without delay.

\section{Description of mortality cases}

The mean age of the seven deaths was 73 years (range, 69-78 years). All mortality cases except one were male. In all cases except VI, amylase-rich peritoneal fluids from drainage tube were identified after postoperative day 7 . Underlying pathologic diagnosis were malignant disease in six cases except in one intraductal papillary mucinous neoplasm (case VI), three cases of bile duct cancer, two cases of ampulla of Vater cancer and one case of pancreas cancer. Laparotomic PJ reconstructions were four patients and laparotomic PG cases were two patients. One laparoscopic PG was included in the mortality cases.

One patient developed hepatorenal syndrome followed by PD was not survived in the observation group. The other reoperation cases were six and the timing of the reoperation was mean 8.5 days (range, 1-14 days) after PD. The factors that led to the decision of reoperation were an anticipating pancreatico-enteric failure by surgeon's decision according to the operation findings and the definite radiologic PEAF. Anticipating failure was the result of intrinsic decision making of surgeon by combining clinical findings of pancreas during surgery and immediate clinical changes of patients. Whereas the radiologic anastomosis failure was defined as the precise description about the disruption of the pancreatico-enteric anastomosis on CT scan: showing the complete or partial 
Table 4. Comparison between three subgroups of PEAF group classified according to management method $(n=78)$

\begin{tabular}{|c|c|c|c|c|}
\hline Characteristic & 0 group $(n=50)$ & I group $(n=15)$ & $S$ group $(n=13)$ & P-value \\
\hline Age (yr) & $61.7 \pm 12.7$ & $59.7 \pm 10.5$ & $65.6 \pm 15.1$ & 0.799 \\
\hline Sex (male:female) & $34: 16$ & $11: 4$ & $10: 3$ & 0.792 \\
\hline \multicolumn{5}{|l|}{ ASA class } \\
\hline । & 6 & 2 & 2 & 0.947 \\
\hline$\|$ & 41 & 10 & 10 & 0.452 \\
\hline$\| I I \& I V$ & 3 & 3 & 1 & 0.251 \\
\hline Type of pancreatico-enteric anastomosis & & & & 0.306 \\
\hline Laparoscopic & 8 & 2 & 3 & 0.773 \\
\hline PJ & 1 & 0 & 0 & 0.756 \\
\hline$P G$ & 7 & 2 & 3 & 0.703 \\
\hline Laparotomic & 42 & 13 & 10 & 0.773 \\
\hline PJ & 22 & 11 & 8 & 0.109 \\
\hline$P G$ & 20 & 2 & 2 & 0.063 \\
\hline Pathology & & & & 0.508 \\
\hline Benign ${ }^{\text {a) }}$ & 11 & 5 & 2 & \\
\hline Malignant & 39 & 10 & 11 & \\
\hline Pancreas cancer & 8 & 3 & 3 & 0.819 \\
\hline Bile duct cancer & 22 & 5 & 3 & 0.352 \\
\hline Ampulla of Vater cancer & 7 & 1 & 4 & 0.195 \\
\hline Pancreatic NET & 2 & 1 & 1 & 0.829 \\
\hline Mortality & 1 & 0 & 6 & $<0.001$ \\
\hline Procedures (day)b) & - & $17(6-34)^{c)}$ & $12.2(1-27)^{c)}$ & \\
\hline
\end{tabular}

Values are presented as mean \pm standard deviation or number.

PEAF, pancreatico-enteric anastomosis failure; 0 group, observation group; I group, intervention group; S group, surgery group; ASA, American Society of Anesthesiologists; PJ, pancreaticojejunostomy; PG, pancreaticogastrostomy; NET, neuroendocrine tumor.

a) Benign disease: pancreatic cystic neoplasm, pancreatitis, pancreas injury and duodenal tumor, duodenal perforation, periampullary adenoma, etc. ${ }^{\text {b) Postopera- }}$ tive day of procedure performed, mean (range). ') Even if the procedure was performed in one patient, each procedure was separately counted.

Table 5. Procedures performed in surgery group of PEAF group after pancreaticoduodenectomy $y^{\mathrm{a}}$

\begin{tabular}{lccc} 
Surgical procedure & $\begin{array}{c}\text { No. of procedure } \\
(\mathrm{n}=\mathbf{1 4})^{\mathrm{b})}\end{array}$ & $\begin{array}{c}\text { Time between diagnosis and } \\
\text { relaparotomy, mean (range), day }\end{array}$ & $\begin{array}{c}\text { Mortality } \\
\text { Completion total pancreatectomy with splenectomy }\end{array}$ \\
Completion total pancreatectomy without splenectomy & 4 & $11.4(1-26)$ & 4 \\
PG direct repair & 1 & 7 & 2 \\
R-Y PJ & 1 & 11 & 0 \\
\hline
\end{tabular}

PEAF, pancreatico-enteric anastomosis failure; PG, pancreaticogastrostomy; R-Y, Roux-en-Y anastomosis; PJ, pancreaticojejunostomy.

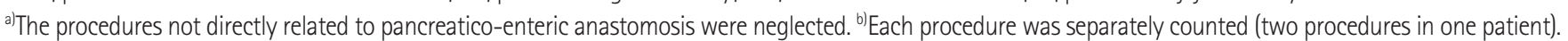

anastomotic breakdown with an associated local fluid collection, based on amylase rich fluid with clinical features (fever, worsening of abdominal pain, etc.). The most common reoperation procedure was total pancreatectomy with splenectomy $(n=8)$. The major cause of death was liver failure, sepsis, and the average death time was 31 days (range, 7-43 days) after PD (Table 6).

\section{DISCUSSION}

The definition and classification of pancreatic fistula after PD re- section has been various, however, most of them have since followed the definition and classification proposed by the International Study Group on Pancreatic Fistula (ISGPF) in 2005 and 2016. According to the guideline of ISGPF, pancreatic fistula defined as postoperative pancreatic fistula and the persistent drainage of amylase-rich fluid: greater than three times the upper normal serum value, during the postoperative course especially on or after postoperative day 3 . Also it was proposed that the grading system based on this definition, considering clinical condition, radiologic findings, reoperation, and infection $[1,3,14]$. 
Table 6. Seven mortality cases related to PEAF after PD resection

\begin{tabular}{|c|c|c|c|c|c|c|c|c|c|}
\hline Case & Age (yr) & Sex & $\begin{array}{l}\text { Amylase } \\
(\mathrm{IU} / \mathrm{L})^{\mathrm{a})}\end{array}$ & $\begin{array}{l}\text { Time } \\
(\text { day })^{b)}\end{array}$ & Diagnosis & $\begin{array}{l}\text { Time } \\
(\text { day })^{c)}\end{array}$ & Reason for re-OP & Cause of death & $\begin{array}{l}\text { Time } \\
(\text { day })^{d)}\end{array}$ \\
\hline 1 & 69 & M & 28,280 & 9 & AoV ca. & 14 & Anticipating PJ failure & Liver failure & 32 \\
\hline$\|$ & 74 & $\mathrm{~F}$ & 12,260 & 12 & BD ca. & 12 & Anticipating PJ failure & Liver failure & 33 \\
\hline$\|\left.\right|^{e)}$ & 74 & $M$ & 11,100 & 12 & Pancreas ca. & - & - & HRS & 42 \\
\hline IV & 72 & M & 28,400 & 6 & BD ca. & 7 & Radiologic PJ disruption & Sepsis & 7 \\
\hline$V^{f)}$ & 68 & M & 98,600 & 8 & AoV ca. & 8 & Radiologic PG disruption & Bleeding & 43 \\
\hline$V^{(9)}$ & 78 & M & - & - & IPMN & 1 & Anticipating PJ failure & ARDS & 37 \\
\hline VII & 76 & $M$ & 42,100 & 7 & BD ca. & 9 & Radiologic PG disruption & Sepsis & 23 \\
\hline
\end{tabular}

PEAF, pancreatico-enteric anastomosis failure; $\mathrm{PD}$, pancreaticoduodenectomy; OP, operation; $\mathrm{M}$, male; $\mathrm{F}$, female; AoV, ampulla of Vater; ca., cancer; PJ, pancreaticojejunostomy; BD, biliary duct; HRS, hepatorenal syndrome; PG, pancreaticogastrostomy; IPMN, intraductal papillary mucinous neoplasm; ARDS, acute respiratory distress syndrome.

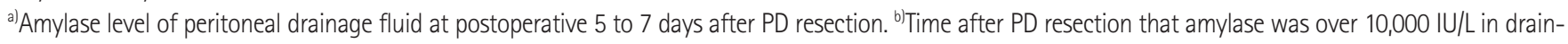
age fluid: the date shown is the postoperative date from PD resection. ${ }^{c}$ When the reoperation was performed after PD resection: the date shown is the postoperative date from $\mathrm{PD}$ resection. ${ }^{\mathrm{d}}$ Time of death after PD resection. ${ }^{\mathrm{e}} \mathrm{Case}$ III was the intervention group (PG failure). ${ }^{\mathrm{ff}} \mathrm{Case} \mathrm{V}$ was a laparoscopic surgery. ${ }^{\mathrm{g}} \mathrm{Case} \mathrm{VI}$ had liver cirrhosis.

However, many criticisms have been provoked for the original strict definition criteria of ISGPF, so it cannot apply to clinical situation practically $[2,15]$. So, the updated classification of pancreatic fistula was proposed by ISPGF in 2016. Unlike 2005 classification, which all cases defined as fistula by abnormal amylase level, the 2016 classification distinguished the true fistula from a biochemical leak that the amylase level was elevated without related clinical features or signs. The true pancreatic fistula cases with amylase level elevation and clinical manifestation or patient condition change, or management change was classified as grade $\mathrm{B}$ or $\mathrm{C}$ according to reoperation and organ failure.

Nevertheless, this updated classification still does not specify a detailed classification of elevated level and periods of amylase. As a result, the definition of ISGPF might not acceptable to all cases and the grading system seems to complex, and intermingled furthermore includes multiple subjective criteria [16]. So, we categorize patients simply according to the definitive PEAF base on radiologic findings and clinical condition related to clear inflammatory sign. And the concept of the pancreatic fistula is ambiguous and extensive, so we use the term PEAF to define the concept of leakage and failure of the pancreatic anastomosis site.

The amylase level of drainage fluid about up to 3 days postoperatively is of less clinical importance, because of the many cases that are elevated by normal postoperative reactions and the most PEAF occurred in 5 days PD [17]. However, amylase-rich peritoneal fluid should be associated with PEAF obviously after 5 days postoperatively. Thus, we defined as previously described, the presence of rich amylase in operatively placed or percutaneous placed drainage fluid at postoperative days 5 to 7 . For simple application of these criteria, we set the reference value at over 10,000 IU/L. If the position of drain catheter is not proper or changed, reliability of the peritoneal fluid analysis would be disappointed. Therefore, we performed CT scan on postoperative 5 to 7 days routinely, and correlates patient's clinical condition whether or not the inflammatory sign focused on the peritoneal irritation findings and or vital sign.

In our series, male sex was vulnerable to PEAF and it was also noted in other reports [18,19]. PEAF risk was correlated with pancreatic fatty infiltration. The risk of pancreatic fatty infiltration was correlated with visceral fat, in general, visceral fat was higher in men than in women. The reason of this sexual difference of fatty infiltration, male sex is predictive factors for pancreatic fistula [20].

With aging society, surgical candidates of the PD already expanded to septuagenarian and octogenarian. It seems to the one of the cause of increasing surgical risk after PD. Inevitable comorbidity was accompanied with aging. It might affect surgical outcome adversely. Therefore, selection of the patient emphasized especially in the major surgery such as PD [19]. Because once complication occurs in the old age group, the opportunity of recovery is poor. In our series age of most of mortality was seventies.

In general PG was believed in terms of anastomotic security superior to PJ of PD. PJ and PG are the most commonly used methods among various pancreatico-enteric anastomosis methods proposed to prevent PEAF. PJ includes variable method for anastomosis of pancreatic remnant and jejunum as end-to-end (dunking method), end-to-side, end-to-side duct to mucosa anastomosis, etc. [21]. It has superior vascularity and mobility of jejunum. PG is the anastomosis method of pancreatic stump to posterior wall of stomach. And this has advantages following as: (1) possible tension free anastomosis; (2) can avoid traction effect on anastomosis be- 
cause a long jejunal loop with its retained secretion; (3) gastric wall provides excellent blood supply to the anastomosis; (4) easy monitoring of duct patency by nasogastric amylase estimation [22].

Although several studies have suggested that PG is less likely to develop PEAF than PJ, in contrast, a number of studies have reported that there is no significant difference between the two methods $[7,8,23]$. In general, there were many results that PG was superior to PJ, and in our study, it seems that the above benefits are reflected in similar results. We also prefer PG in case of soft pancreas during the PD, but it is not definite factor to decide pancreatico-enteric anastomosis method. Other factors influencing whether $\mathrm{PG}$ or PJ is patient's age, if the patient is younger, $\mathrm{PJ}$ is preferable in our institute. As noted earlier, one of the disadvantageous long term outcomes of PG might endocrine derangement [24]. So endocrine concern is the prefer $\mathrm{PJ}$ of the young age group who has benign disease for the PD.

In this study, there was no difference in the PEAF incidence according to the pancreatico-enteric anastomosis. But the interpretation should be made carefully because there might have selection bias considering pancreas stiffness which is very subjective [25]. It is deducible in this study, in the pancreatic adenocarcinoma, PEAF incidence was strikingly low $(\mathrm{P}<0.001)$. Hard pancreas is commonly coinciding finding and it is not soft nature guarantee a bit pancreatico-enteric anastomosis, fortunately. On the contrary, pancreas of all the other malignancy besides pancreas cancer such as bile duct cancer, ampulla of Vater cancer and neuroendocrine cancer seems to have soft consistency. In our series, PD for pancreas neuroendocrine tumor shows $44 \%$ PEAF rate (4/9). We need to investigate additional possible risk factors other than pancreas stiffness issue [22].

As in this study, supportive treatment is sufficient to improve PEAF in spite of peritoneal high amylase level (over 10,000 IU/L). However, in that situation adequate drainage should be established in the PD procedure. Otherwise radiologic interventional drainage tube insertion should be essential. Ultrasonic or fluorescent guided transabdominal drainage catheterization can avoid risky reoperation. In the case of failed radiologic intervention or supportive treatment, timely operative strategy should be involved. In some situation of PD, pancreatico-enteric anastomosis status was not satisfactory to the surgeon, that are the situation like the poor jejunal vascular supply and the poor suture handling due to very soft pancreas. Like that case, prophylactic total pancreatectomy might have better outcome than PEAF [26]. But it is not an easy decision because of the relative poor quality of life in the patient after recovery such as lifelong insulin requirement. Outcome of reoperation for the PEAF after PD was significantly affected by the patient general condition at the moment. If you do the reoperation timely, you can preserve spleen in some case. In this study, we can preserve spleen in two reoperation patients. However, the spleen preserving does not seem to have benefit in the patient outcome. In the case of insecure pancreatico-enteric anastomosis, even with the adequate drainage tube maintenance PEAF might cause catastrophic clinical result such as autodigestion of peripancreatic soft tissue and vessels. Omen for PEAF resultant catastrophe is bleeding from the drain site. Generally angiographic embolization to the bleeding peripancreatic vessels cannot rescue the patient in that tough situation. Without elimination of the underlying PEAF, angiographic attempt further disrupts the blood supply necessary to heal the lesion. So, early surgical intervention was essential but unfortunately their clear criteria establishing is extremely difficult. But interval of the initial PD and reoperation was within 14 days in all of our mortality cases after reoperation. We had to look back the PD indication again and timing of the surgical intervention had to be reanalyzed again case by case basis. Fortunately, most of PEAF patient was successfully managed with supportive care and radiologic interventional treatment in our series. The choice between the PG and PJ had to be concluded but in the risk group such as very soft nature in consistency, randomization study might have the ethical problem. We had to carefully select the patient not suitable for the $\mathrm{PD}$ and minimally invasive approach in that group other than PD.

PD is still challenging procedure because of PEAF. Male sex and periampullary cancer besides pancreatic cancer has the risk of pancreas anastomotic failure. Adequate drainage catheter indwelling during the PD or postoperative radiological intervention can effectively manage the PEAF without interventional treatment.

\section{CONFLICT OF INTEREST}

No potential conflict of interest relevant to this article was reported.

\section{REFERENCES}

1. Bassi C, Dervenis C, Butturini G, Fingerhut A, Yeo C, Izbicki J, et al. Postoperative pancreatic fistula: an international study group (ISGPF) definition. Surgery 2005;138:8-13.

2. DeOliveira ML, Winter JM, Schafer M, Cunningham SC, Cameron JL, Yeo CJ, et al. Assessment of complications after pancreatic surgery: a novel grading system applied to 633 patients undergoing pancreaticoduodenectomy. Ann Surg 2006;244:931-7.

3. Bassi C, Marchegiani G, Dervenis C, Sarr M, Abu Hilal M, Adham M, et al. The 2016 update of the International Study Group (ISGPS) definition and grading of postoperative pancreatic fistula: 11 years after. Surgery 2017;161:584-91.

4. Trede M, Schwall G. The complications of pancreatectomy. Ann 
Surg 1988;207:39-47.

5. Miedema BW, Sarr MG, van Heerden JA, Nagorney DM, Mcllrath DC, Ilstrup D. Complications following pancreaticoduodenectomy. Current management. Arch Surg 1992;127:945-9.

6. Morris DM, Ford RS. Pancreaticogastrostomy: preferred reconstruction for Whipple resection. J Surg Res 1993;54:122-5.

7. Yeo CJ, Cameron JL, Maher MM, Sauter PK, Zahurak ML, Talamini MA, et al. A prospective randomized trial of pancreaticogastrostomy versus pancreaticojejunostomy after pancreaticoduodenectomy. Ann Surg 1995;222:580-8.

8. Wente MN, Shrikhande SV, Muller MW, Diener MK, Seiler CM, Friess $\mathrm{H}$, et al. Pancreaticojejunostomy versus pancreaticogastrostomy: systematic review and meta-analysis. Am J Surg 2007;193:17183.

9. Alexakis N, Sutton R, Neoptolemos JP. Surgical treatment of pancreatic fistula. Dig Surg 2004;21:262-74.

10. Aranha GV, Aaron JM, Shoup M, Pickleman J. Current management of pancreatic fistula after pancreaticoduodenectomy. Surgery 2006;140:561-8.

11. Batignani G, Fratini G, Zuckermann M, Bianchini E, Tonelli F. Comparison of Wirsung-jejunal duct-to-mucosa and dunking technique for pancreatojejunostomy after pancreatoduodenectomy. Hepatobiliary Pancreat Dis Int 2005;4:450-5.

12. Kim EY, You YK, Kim DG, Hong TH. A simple pancreaticojejunostomy technique for hard pancreases using only two transpancreatic sutures with buttresses: a comparison with the previous pancreaticogastrostomy and dunking methods. Ann Surg Treat Res 2016;90:64-71.

13. Hong TH, Youn YC, You YK, Kim DG. An easy and secure pancreaticogastrostomy after pancreaticoduodenectomy: transpancreatic suture with a buttress method through an anterior gastrotomy. J Korean Surg Soc 2011;81:332-8.

14. Kim WS, Choi DW, Choi SH, Heo JS, Kim MJ, Song SC, et al. Clinical validation of the ISGPF classification and the risk factors of pancreatic fistula formation following duct-to-mucosa pancreaticojejunostomy by one surgeon at a single center. J Gastrointest Surg 2011;15:2187-92.

15. Shrikhande SV, D'Souza MA. Pancreatic fistula after pancreatectomy: evolving definitions, preventive strategies and modern management. World J Gastroenterol 2008;14:5789-96.
16. Strasberg SM, Linehan DC, Clavien PA, Barkun JS. Proposal for definition and severity grading of pancreatic anastomosis failure and pancreatic occlusion failure. Surgery 2007;141:420-6.

17. van Berge Henegouwen MI, De Wit LT, Van Gulik TM, Obertop H, Gouma DJ. Incidence, risk factors, and treatment of pancreatic leakage after pancreaticoduodenectomy: drainage versus resection of the pancreatic remnant. J Am Coll Surg 1997;185:18-24.

18. Machado NO. Pancreatic fistula after pancreatectomy: definitions, risk factors, preventive measures, and management-review. Int J Surg Oncol 2012;2012:602478.

19. Aoki S, Miyata H, Konno H, Gotoh M, Motoi F, Kumamaru H, et al. Risk factors of serious postoperative complications after pancreaticoduodenectomy and risk calculators for predicting postoperative complications: a nationwide study of 17,564 patients in Japan. J Hepatobiliary Pancreat Sci 2017;24:243-51.

20. Mathur A, Pitt HA, Marine M, Saxena R, Schmidt CM, Howard TJ, et al. Fatty pancreas: a factor in postoperative pancreatic fistula. Ann Surg 2007;246:1058-64.

21. Hosotani R, Doi R, Imamura M. Duct-to-mucosa pancreaticojejunostomy reduces the risk of pancreatic leakage after pancreatoduodenectomy. World J Surg 2002;26:99-104.

22. Seetharam P, Rodrigues GS. Postoperative pancreatic fistula: a surgeon's nightmare an insight with a detailed literature review. JOP 2015;16:115-24.

23. Topal B, Fieuws S, Aerts R, Weerts J, Feryn T, Roeyen G, et al. Pancreaticojejunostomy versus pancreaticogastrostomy reconstruction after pancreaticoduodenectomy for pancreatic or periampullary tumours: a multicentre randomised trial. Lancet Oncol 2013;14:655-62.

24. Jang JY, Kim SW, Park SJ, Park YH. Comparison of the functional outcome after pylorus-preserving pancreatoduodenectomy: pancreatogastrostomy and pancreatojejunostomy. World J Surg 2002;26:366-71.

25. Sato N, Yamaguchi K, Chijiiwa K, Tanaka M. Risk analysis of pancreatic fistula after pancreatic head resection. Arch Surg 1998;133:10948.

26. Gueroult S, Parc Y, Duron F, Paye F, Parc R. Completion pancreatectomy for postoperative peritonitis after pancreaticoduodenectomy: early and late outcome. Arch Surg 2004;139:16-9. 